\title{
Nanomedicines Design: Approaches towards the Imaging and Therapy of Brain Tumours
}

Matthew Chapman and Sofia I. Pascu*

Chemistry Department, University of Bath, Claverton Down, Bath, UK

\begin{abstract}
Nanotechnology has potential to revolutionize diagnosis and therapy of diseases in the central nervous system through nanotheranostics. This simply states that a nanocarrier can be designed in an 'all-in-one' approach to facilitate both the functions of a diagnostic and a therapeutic drug. There are many challenges that must be overcome for these nanocarriers to become a successful design, but if resolved nanocarriers could pave the way for personalised medicine. This review highlights several new ideas behind nanotheranostics design and testing with a particular focus on cancerous tumours in the brain.
\end{abstract}

Keywords: Nanotheranostics; Nanocarrier; Imaging; Therapy; Brain tumour; Carbon nanotube

\section{Introduction}

'Nano' is derived the Greek word for 'dwarf' and is used to describe structures and objects that are sized within the scale of 1 to 100 nanometres $\left(\mathrm{x} 10^{-9} \mathrm{~m}\right)[1]$. Nanotechnology can be defined as the application of structures of this size [1]. It is rapidly becoming a reality that nanotechnology allows the creation of new materials for a wide array of purposes ranging from medical, electronic, biomaterials, to even energy production.

The medical applications of nanotechnology are referred to as Nanomedicine. This is a wide and exciting field with almost boundless possibilities, with the possibility for nanotechnology being used for applications ranging from the use of nanomaterials, nanoelectronic biosensors and has a wide range of future applications of molecular nanotechnology that could be explored. One of these applications is the use of targeted nanocarriers to transport drugs to the target site of the disease where they can be released. There are many problems that need to be overcome, but this is an area that shows enormous potential.

This review focuses on diseases that reside in the central nervous system (CNS), in particular the brain, and how nanocarriers can be designed and used to treat and diagnose them. This will be with particular reference to cancerous tumours. Currently the method for treating and diagnosing diseases in the brain is to use free drugs or radiolabelled molecules to get to the target site and fulfil its function. The imaging and therapy with small molecules have many disadvantages that could be addressed via encapsulation, or attachment, methods in order to increase their performance, including not being prematurely interacting with the biological environment or being prematurely removed from the body. Therapy and imaging with small molecules also suffer from issues of entering the target site, as the brain is extremely well protected from toxic molecules including drugs and imaging drugs. For this reason nanocarriers of correct physicochemical characteristics are currently being strongly considered to address these issues. This review will explore the problems associated with drug delivery into the brain and will explain the possible avenues that could be explored to resolve them, and review their potential for using nanodimensional objects for the brain targeting.

One exciting prospect that has recently emerged is the idea of Nanotheranostics, which is emerging from the synergy of the two areas mentioned: diagnostics and therapy using nanodimensional objects. The vision for Nanotheranostics is that nanocarriers can be designed that will serve a dual purpose, allowing both treatment and diagnosis to be contained in an 'all-in-one' package. The nanocarriers will be designed to target a certain disease. These nanocarriers will also have a probe inserted into their design to allow them to be tracked in the body. Therefore if the nanocarriers accumulate in one place, i.e. the target site of the disease, then this will allow doctors to further investigate this area to confirm that the nanocarriers are reacting to diseased cells. These nanocarriers will also carry a drug that will be released if the nanocarrier binds to the cell, starting the therapy of the disease making the treatment of the diagnosis immediate and saving valuable time.

This field is still in its infancy, and although it has many applications only a few are currently being explored. The most exciting area is the investigation into the use of these nanocarriers for the diagnosis and treatment of cancerous tumours in the brain [2].

The two different ways of targeting nanocarriers are via processes called active targeting or passive targeting. These utilise different systems to gain access to the tumorous cells, but unfortunately the precise design requirements for achieving successful active or passive transport into cancerous tumours in the brain has not been discovered. These targeting methods have been successfully used for other tumours site.

A passive targeting nanocarrier does not have any additions to its structure to direct it to the desired diseased target site; it instead relies on its properties (charge, size) to arrive at the correct location in the body. An active targeting nanocarrier, on the other hand, does possess ligands attached onto its structure that guide it to the correct location, and should act like a key to let it gain access to the target cell. This is represented in Figure 1. The idea of active targeting is still an idea in early development and still has many issues to overcome.

Passive targeting works through faults that develop in the blood

*Corresponding author: Sofia I Pascu, Chemistry Department, University of Bath, Claverton Down, Bath, UK, E-mail: s.pascu@bath.ac.uk

Received February 09, 2012; Accepted March 08, 2012; Published March 11 2012

Citation: Chapman M, Pascu SI (2012) Nanomedicines Design: Approaches towards the Imaging and Therapy of Brain Tumours. J Nanomedic Nanotechnol S4:006. doi:10.4172/2157-7439.S4-006

Copyright: (c) 2012 Chapman M, et al. This is an open-access article distributed under the terms of the Creative Commons Attribution License, which permits unrestricted use, distribution, and reproduction in any medium, provided the original author and source are credited. 


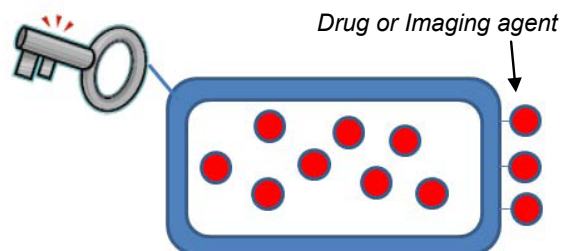

Active Targeting Nanocarrier

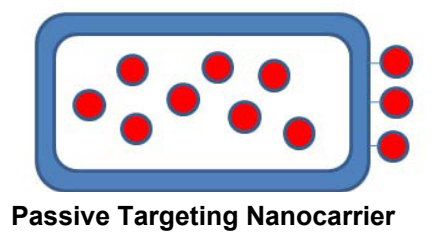

Figure 1: Schematic representation of active and passive targeting. The ligands attached to the active targeting molecule (red) to guide it to the target site, represented by the 'key'. The diagram also reveals the two methods for storing drug molecules and imaging agents, either encapsulated within the nano-carrier or covalently linked to the external nano-structure.

vessels that surround a diseased site, an example of this is in tumorous cells. Tumours require a blood supply to survive, and use angiogenesis to create new blood vessels from existing ones. The majority of tumours develop leaky blood vessels and shown poor lymphatic drainage due to rapid and defective angiogenesis [2]. Free drugs diffuse non-specifically into these cells but correctly designed nanocarriers escape into the tumour cells through leaky blood vessels via the enhanced permeability and retention effect [3]. This effect only affects molecules of a certain size $(200-400 \mathrm{~nm})$ and these molecules as a result tend to accumulate in tumour cells [4-6]. In this example, nanocarriers [3] are then retained by the dysfunctional lymphatic drainage in tumours which allows the drugs to be released near the tumour site.

Passive targeting suffers from several limitations. Due to the random nature of the process the nanocarriers may accumulate in one section of the tumour preventing the drugs to diffuse equally across the tumour [2]. This is a lack on of control with this method means that the nanocarriers often induce the function efflux proteins, which remove the nanocarrier from the cell $[7,8]$ This effect will be investigated in detail in the process of this review. Passive targeting is further limited as tumours don't show the same permeability throughout their structure, therefore the enhanced permeability and retention effect becomes less effective [9].

Active targeting nanocarriers would solve these problems and could provide a general solution for drug delivery to the CNS, including tumorous cells in the brain. The design of these actively targeted nanocarriers that target drugs to the diseased site, without affecting healthy tissues, whilst avoiding side effects is currently a matter of intense research as there are still many problems that must be overcome.

\section{Problem}

Many challenges related to the drug design and drug delivery route must be resolved before actively targeted nanocarriers can be a viable route for drugs to the brain to diagnose or cure diseases of the CNS including tumours in the brain in a selective way. This is currently an area of intense research.
Some of these same problems have already been solved by simple chemicals which target brain (e.g. glucose, morphine) and are recently being extended to nanomaterials, but one problem called the blood brain barrier (BBB) is the main bottleneck to the design for achieving successful active or passive transport. A key issue related to drug design is that drugs and nanocarriers must have a long circulation time in blood to allow the active targeting to take place. The biocompatibility of the drug and the ability to bypass the liver are the most important factors that correlate to circulation time [10]. Biocompatibility allows the drugs and nanocarriers to avoid being detected as a foreign body and destroyed by body's natural defences, i.e. phagocytosis. The ability to bypass the liver prevents the drug or nanocarrier to be removed from the body before it is able mission is complete. These attributes can be given to nanocarriers by correct design and will be discussed in the 'Probe Design toward a Targeted Delivery Solution' section of this review. The solution to crossing $\mathrm{BBB}$ and the reasons behind the current issues for CNS drugs are explored below.

\section{Blood brain barrier (BBB)}

The function of the BBB is to protect the CNS from toxic molecules that, for example, may be ingested in food.11 In addition to of this function the $\mathrm{BBB}$ serves as a barrier to drugs for treatment of diseases of the CNS. This means that only a very small selection of drugs, of correct liphophylicity and with small molecular weights manage to enter the CNS through the BBB. No drugs with large molecular weights enter the CNS through the BBB, except for a small minority of natural proteins, e.g. insulin, that have specific pathways designed to carry them across the $\mathrm{BBB}$. The current challenge is to devise new nanodimensional targeting drugs having the ability to cross BBB.

The structure of the Blood Brain Barrier involves endothelial cells that form the walls of the capillaries in the brain. These endothelial cells are surrounded by tight junctions and lack the large pores that are generally found in capillary walls (Figure 2). Tight junctions are formed by two closely associated cells, there membranes join together to form a virtually impermeable barrier to most substances. Tight junctions prevent many polar solutes from entering the CNS [11]. Lipid soluble molecules can cross the BBB through the endothelial cells, but this is regulated by ATP requiring efflux transporters that selectively remove

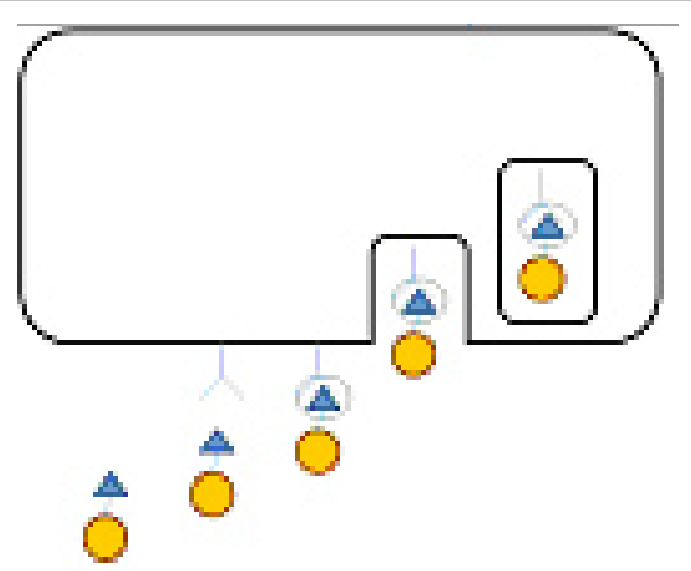

Figure 2: A schematic representation of a cell and its nucleus: Diagram shows of endocyotic event caused by a ligand on a nanocarrier binding to a receptor on the surface of the cell. The resulting cluster, along with the macromolecule, 'pinches off' into a vesicle which is then engulfed into the cell, moved across and exocytosed on the opposite plasma membrane. 
molecules from the cell. This ensures that only selected molecules with specific physical and thus reactivity pathways can cross the BBB. The transport across the Blood Brain Barrier is a matter of intensive research. The brain is the most energy consuming organ of the body and has developed systems to transport the wide variety of required substances across the BBB. The use of these systems will be key to the success of using nanocarriers for active targeting of drugs to cure diseases of the CNS.

Passive diffusion: A wide range of small lipid soluble substances can passively diffuse through the cell membrane and cross the endothelium [12]. It is through this pathway that most current CNS drugs cross the BBB. These drugs must possess a number of physicochemical properties. This has been a major area of research as it is hoped that once the properties required are defined more successful CNS drugs can be designed. It has been shown that molecules of a molecular weight greater than 450 Daltons will not be able to cross the BBB using this pathway [13]. This makes the design of effective CNS drugs extremely difficult. Lipid solubility is another important physicochemical factor. It has been shown that the more lipid soluble a drug, the greater the probability it will cross the BBB. This has been experimentally shown to be determined by the $\log \mathrm{D}$ octanol/buffer partition coefficient at $\mathrm{pH}$ $7.4[14]$.

In addition, there is a wide range of other factors that also restrict the BBB permeability. These are not an absolute indication of BBB permeability as CNS drugs have been designed that do not possess these properties. Therefore, a defined set of rules for $\mathrm{BBB}$ permeability may not be the best way forward for the design of new drugs capable of targeting the CNS. A key issue is that efflux transporters prevent many CNS drugs from entering the BBB via passive diffusion.

ABC transporter efflux: These transporters are ATP-dependant membrane glycoproteins that actively remove molecules as they diffuse through the membrane or from the cytoplasm. The design of the drug to maximise its $\mathrm{BBB}$ penetration, by increasing the lipid solubility, is often counter-productive as this increase can lead to an increased likelihood of becoming effluxed by one of these transporters [15-19]. The most important of these are P-glycoprotein (Pgp), the Multidrug Resistanceassociated Proteins (MRP 1-5*) and Breast Cancer Resistance Protein (BCRP) [16,20,21].

In addition to designing compounds that have the physicochemical properties needed to enable passive diffusion across the BBB, it is now required that these compounds are poor substrates for these efflux transporters. This fact adds to the increasing difficulty in designing a successful CNS drug. Considering that using this method only a relatively small proportion of the drug that is administered crosses the $\mathrm{BBB}$ other methods must be considered. This is the main reason why actively targeted nanocarriers could hold the answer, as many druglike, active molecules can be delivered to target in an 'all-in one' design whilst keeping the actual dose of the overall carrier extremely small.

Carrier mediated influx via solute carriers: Carrier mediated influx via solute carriers may offer the possibility of drugs crossing the $\mathrm{BBB}$ via active transport. Water-soluble carriers transport vital metabolites such as glucose, other sugars, lactate, nucleosides, fatty acids and vitamins into the brain [11].

These solute carriers may be:

- Bidirectional: determined by the substrate concentration gradient
- Unidirectional: either into or out of the cell depending on the substrate

- Or, involving an exchange of one substrate for another, often driven by an ion gradient [11].

In order to utilize this pathway, drugs must be designed to closely resemble these nutrients. This can be closely linked to the idea of the Trojan horse in Greek mythology: the method involves 'tricking' the solute carriers into thinking the drug is another molecule.

This method would increase the administered dose of the drug that enters the CNS as the drug is now actively transported across the BBB. However, this puts even tighter restrictions on the already complex drug design but opens up interesting avenues for synthetic chemists and pharmacologists.

Transcytosis (Receptor mediated and adsorptive mediated): Transcytosis transports macromolecules across the $\mathrm{BBB}$ and into the CNS. The majority of large molecules are prevented from entering the CNS but this specific pathway has developed to allow the transport of necessary large molecules and complexes [11].

This pathway is the best candidate for allowing nanocarriers to cross the $\mathrm{BBB}$ as it is the only pathway that allows for the relatively large molecular weights of nanocarriers.

In Receptor Mediated Transcytosis (RMT) specific receptors on the surface of the endothelial cell bind to specific ligands attached to macromolecules. This triggers an endocyotic event where the receptor and ligand bind forming a cluster. The resulting cluster, along with the macromolecule, 'pinches off' into a vesicle (Figure 2) [11]. This vesicle is then engulfed into the cell, moved across and exocytosed on the opposite plasma membrane [11]. In Adsorptive Mediated Transcytosis (AMT) there must be an excessive positive charge on the molecule [22] When this interacts with the cell surface binding sites, a similar process occurs.

RMT is the most suited pathway for larger drugs such as those supported on nanocarriers to be delivered to cells as it could allow nanocarriers to cross the BBB if a specific ligand is found and attached to the outside of the probe (Figure 3 ).

Problems may arise if the ligand is not specific to the BBB as is recognised by receptors elsewhere as the nanocarrier could be taken up into other cells in the body causing side effects. Ideally the ligand used

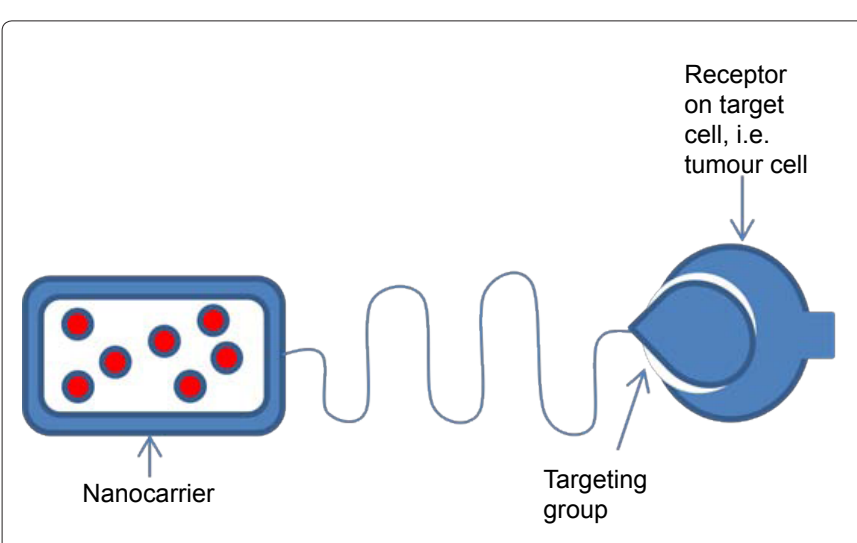

Figure 3: T Schematic representation of a nanocarrier incorporating an imaging or therapeutic molecule ' $M$ ' with an attached ligand. If the correct ligand is added and it comes in contact with the correct ligand, as shown then an endocyotic event will be triggered. 


\begin{tabular}{|l|l|}
\hline Ligands & Receptors \\
\hline Ga, In, Fe & Transferrin, Lactoferrin \\
\hline TI, K & Na, K ATPase \\
\hline Glucose, FDG & Direct G-Protein Activation \\
\hline Substance P & Amino acid \\
\hline a-methyltyrosine & Estrogen \\
\hline Estrogen, analogs, tamoxifen & Sigma \\
\hline Lodobenzamides & Melanocyte \\
\hline For more detailed information see reference [23]. \\
\hline
\end{tabular}

Table 1: Selected receptors, and their corresponding ligands that are over expressed in tumour cells.

should correspond to a receptor that is over-expressed at the target area to improve active targeting. This has been an area of much research and Table 1 shows a list of ligands and associated receptors that could be used for tumorous cells. The advantage of using this pathway is the flexibility that it offers as there is not several physicochemical properties that the nanocarrier is required to process. AMT is not as suitable as it lacks the specificity required for nanocarriers.

Leukocytes: Leukocytes cross the BBB via leukocyte extravasation through endothelial cells either close to the tight junctions or via modified tight junctions [11]. This pathway may have the potential to have a great impact on drugs' ability to cross the BBB, but currently not enough is known in this area to take advantage of this pathway in the drug design. Current research involves opening the tight junctions: this could be potentially dangerous as then anything can enter the brain, even harmful substances. If further research is done in this area this pathway could hold the answer, but there will always be an inherent risk in altering the tight junctions of the BBB.

The examination of the pathways through which molecules can cross the BBB has revealed the reasons why current CNS drugs struggle to cross the BBB. It has also shown that the receptor mediated transcytosis (RMT) is the most suitable pathway for nanocarriers to cross. More research must be completed in this area to find the correct receptors to use, but this area does show great potential.

\section{Probe Designed Towards a Targeted Delivery Solution}

To design a probe to take advantage of the RMT pathway to deliver drugs to the site of a cancerous tumour, there are multiple factors that must be taken in to account. We must consider the material used to act as the nanocarrier, the properties of that can be given to that material and how the drug molecule will be attached.

\section{Choice of nanocarriers: selected nanomaterials for drug delivery}

There is a vast amount of research in the field of materials chemistry proposing new materials that are all currently under development for biomedical applications. The only feature in common all these materials have is there high surface to volume ratio, which allows a high density of ligands to be attached. This allows medical chemist to alter the material's properties for the specific function on the nanocarrier. This review will only focus on a few that fit the desired properties for active targeting. In Figure 4 the materials that are discussed in this review are circled in green.

Polymers: Polymeric nanoparticles were the first polymeric nanocarrier studied for drug delivery devices; they were prepared from a block of polymeric material giving the material an increased blood circulation time and a reduced phagocytic uptake [10]. Biodegradable polymers were then incorporated to maximise the tissue compatibility and minimise the toxicity of the material [25]. This basic idea of incorporating biodegradable polymers into the stable material to alter its properties is one of the basic fundamental ideas behind deign of a targeted delivery solution. It has been shown that addition of materials such as poly(ethylene glycol) (PEG) and other bioactive peptides have been proven to minimise uptake in liver cells and result in greater bioavailability [10]. This is a perfect example of the use of this simple technique is how it is possible to increase nanocarriers properties through simple addition of ligands.

Polymer based nanoparticles have since been developed into a more complicated field with a wide and successful range of nanocarriers to include bionanocapusles and solid lipid nanoparticles. Polymeric nanocarriers internalise the drugs giving the material excellent control over the degradation and release of that drug through the properties of the polymer used.

Vesicles: Liposomes are the simplest forms of vesicles and are the most prevalent in this area; in particular for passive targeting. Liposomes are small artificial vesicles that form a globular shape that consists of an aquatic centre encapsulated with amphiphillic phospholipids and a cholesterol bilayer (Figure 5) [26]. Small unilamellar vesicles with a single lipid layer and a diameter of $25-50 \mathrm{~nm}$ are best suited to active targeting due to their smaller size [27]. Liposomes can also increase their blood circulation time by incorporating the polymers PEG [28] This allows them to be particularly effective as nanocarriers. Liposomes do have some potential issues if they are used as the material as it has been shown that there is variation between batch to batch manufacture, making the manufacture of a consistent nanocarrier difficult. Liposomes also suffer from low drug loading efficiency and a poor stability [10] which limits their usage as the ideal candidate for the material for actively targeted nanocarriers thorough BBB.

Micelles: There are two main types of micelle used as nanocarriers;

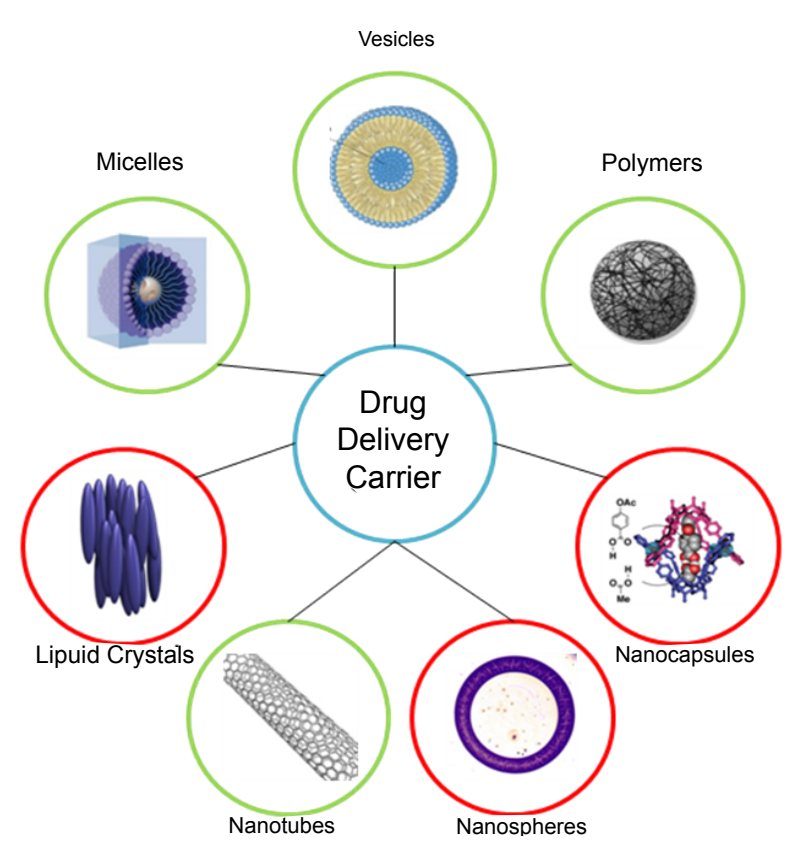

Figure 4: Diagram showing several different materials that are currently under consideration for nanodelivery to the brain, including Micelles, Vesicles, Polymers, Nanocapsules, Nanospheres, Nanotubes and Liquid crystals. Diagram adapted from reference [24]. 


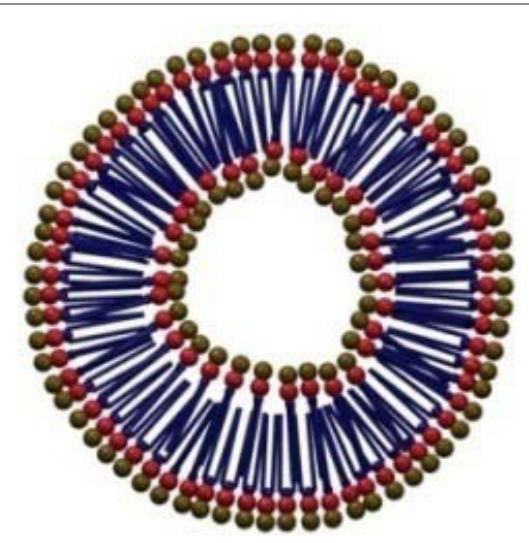

Figure 5: Image of a vesicle.

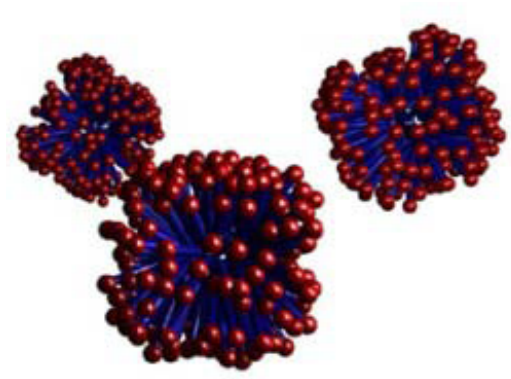

Figure 6: Image of a micelle.

polymeric micelles and phospholipid micelles. Both consist of a hydrophobic core surrounded by hydrophilic surface cells produced by amphiphillic molecules (Figure 6). This alters from a vesicle due to the single layer rather than the bilayer of amphiphillic molecules. This hydrophobic core makes micelles the perfect candidate for when you have poorly soluble drugs, as the drug can be internalized $[29,30]$.

Polymeric micelles are made of polymers and there structure is closest to that of polymeric nanoparticles. Phospholipid micelles structure is closest to that of a vesicle. This means that they both suffer from similar strengths and weaknesses of those materials, but do have an advantage of a smaller size.

Nanotubes: Carbon nanotubes (CNT) are essentially graphene sheets which are rolled up to produce perfect cylinders (Figure 7) [31]. Carbon nanotubes are chemically the most interesting of all the materials considered for the use as nanocarriers, as they give you a degree of control that is not possible with other materials as almost every carbon atom can be functionalised. This allows simple coupling chemistry then to be used to attach any molecule onto the CNT, allowing unlimited variations. This makes CNT the most flexible material that can be used as a nanocarrier.

Due to these limitless possibilities for functionalising the CNT a strong case can be made for this material being used for nanocarriers as it will able to be easily changed to be used for any function that it is needed.

There are two types of carbon nanotubes, single-walled (SWCNTs) and multi-walled (MWCNTs) [31]. SWCNT's are best suited are best suited to being a nanocarrier due to their smaller size, and simpler coupling chemistry. All CNTs have a variable length from only 100$200 \mathrm{~nm}$ to a length of several microns [31]. This adds another factor of control that is possible with CNTs that is not possible with other materials. The diameter of the SWCNT is constantly extremely small as it is only a few nm [31]. Obviously the smaller the length of the CNT the more suitable it is to being a nanocarrier for the applications looked at in this review.

The main drawback of carbon nanotubes that is relevant for use as nanocarriers is that in their non-functionalised form they are almost completely insoluble in all types of solvent [32]. This issue can be resolved by functionalised of the CNT; as it has been shown that with the addition of bioactive peptides on the side walls of the CNT become soluble $[31,33]$. This has only been tested in vivo (in the body) for a limited period, so more research must be conducted before this can be proven to be completely non-toxic. The addition of these groups can be made through the use of simple coupling chemistry. It is through this process that ligands for specific RMT pathways and image active molecules can be added to CNT or any nanocarrier. For this review $\mathrm{CNT}$ will be the focus of this section, although most of the examples shown can be used for other materials.

\section{Simple coupling chemistry to generate biocompatible carbon nanotubes}

To date, there are several methods reported to lead to covalently functionalised nanotubes suitable for biomedical applications. One of the most effective is the addition of 1,3-dipolar cycloaddition of azomethineylides [34]. The unfunctionalised CNTs must be in a

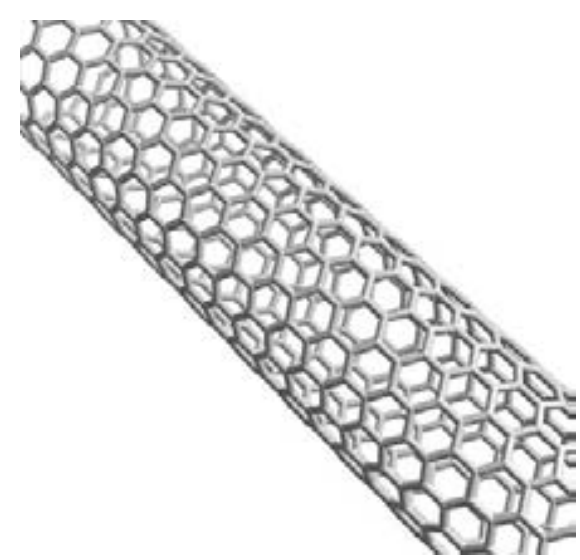

Figure 7: Image of a Carbon Nanotube.

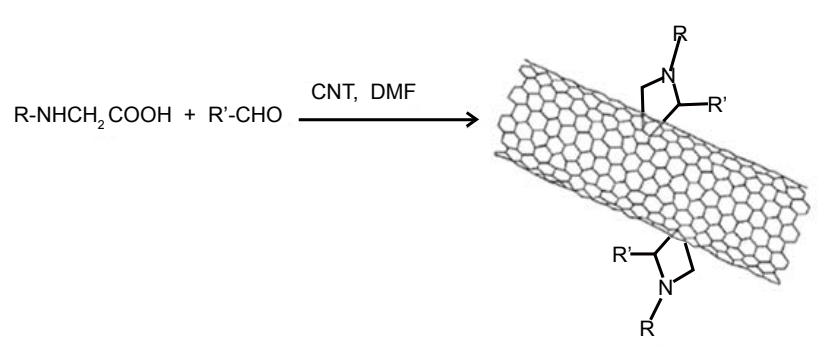

Scheme 1: Addition of 1, 3-dipolar cycloaddition of azomethineylides to functionalise a carbon nanotube. Adapted from reference [31]. 


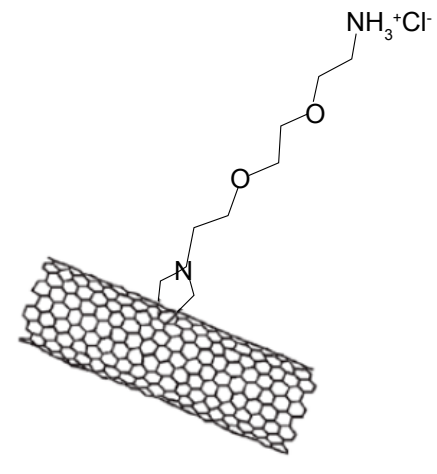

Figure 8: Image of the CNT with an easily functionalized group attached, i.e. $\mathrm{PEG}-\mathrm{NH}_{3}{ }^{+} \mathrm{Cl}$. Adapted from reference[31].

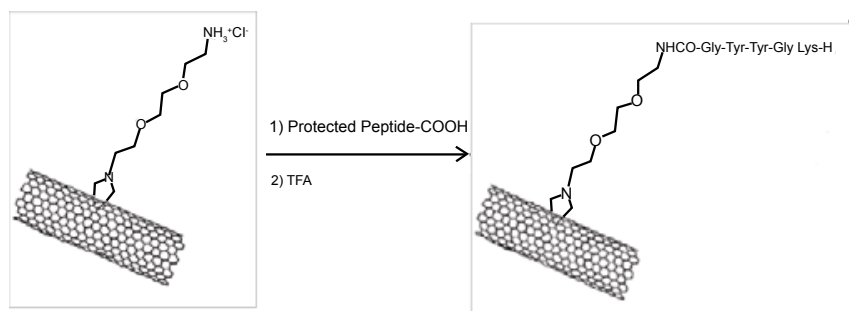

Scheme 2: Addition of bioactive molecules via $-\mathrm{COOH}$ group Adapted from reference [31].

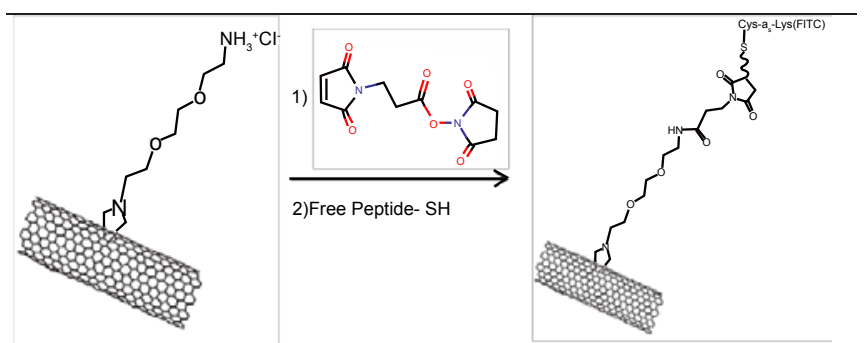

Scheme 3: Addition of Chemical ligand e.g. Fluorescent group via $-\mathrm{SH}$ groups. Adapted from reference [31].

suspension of dimethylformide (DMF) and then a combination of $\mathrm{N}$-substituted glycine and an aldehyde must be added. The reaction scheme is displayed in Scheme 1. The new functionalised groups produced by this reaction will be homogenously distributed all along CNT, allowing multiple different ligands to be added.

The effect of this functionalisation is that it allows the properties of the CNT to be altered by the addition of various ligands through different reactions. This can be achieved by using an accessible functionalised CNT: one such example is shown in Figure 8. This CNT can then be reacted to allow the addition of different ligands through various reactions, some examples are shown in Schemes (2-4). The percentage coverage of each ligand added can be controlled via the limitation of the amount of each ligand and reactant added.

There are many more standard coupling reactions that can be adapted for this purpose many of which highlighted in organic textbook
[35]. There are issues that must be considered when choosing which coupling reactions to use, as a minute amount catalyst and reactants could reside in the CNT system as if these are toxic then could affect their efficiency and biocompatibility. This is an area that will have to be careful researched.

\section{Appending drug-line molecules to Nanocarriers}

In the design of nanocarriers a major consideration is the selection drugs that the nanocarriers are designed to deliver and the method via which they deliver those drugs.

Method of attachment: The drugs can either be covalently linked to the external walls of the nanocarrier or they can become encapsulated into the structure. Drugs that are encapsulated can be either water or lipid soluble. There are many advantages of encapsulation as it prevents the drug from prematurely degrading or prematurely interacting with the biological environment [2]. The disadvantage of encapsulating is that the nanocarrier has to be homogenised for the drugs release meaning that drug release is difficult to control; although this does normally mean the drug is slowly released over time $[2,36]$. Encapsulating drugs could also suffer from a relatively poor drug loading as nanocarriers by definition have a high surface area to volume ratio. This means that a large quantity of the drug can be covalently linked onto the external walls of the nanocarrier.

Drug-like molecules that are covalently linked onto the external walls of the nanocarrier can be only water soluble, because if a lipid soluble drug was added then this would affect the nanocarriers' properties [10]. The advantage of covalently linking drugs onto nanocarriers is the fact that this allows good drug loading and allows the best chance for an external trigger to be used for drug release [10]. This trigger could be a range of environmental conditions, or could possible come from an external source. The disadvantage of covalently linking is that it does allow the drugs to come in contact with the biological environment, but fortunately most drugs are inactive when linked. Drugs can be easily attached through simple coupling chemistry to the external walls, the method for encapsulation can be often much more complex. Overall it depends on the type of drug to determine which method is preferred; although for CNT external linkage could be the preferred method due to their small diameter making the issue of drug loading worse.

Selected chemotherapeutics for brain tumour: There are thousands of drugs that could potentially benefit through the use of nanocarriers, including hundreds that are aimed towards tumorous cells. Chemotherapy drugs designed to rapidly reproducing cells, both cancerous and healthy, causing many side effects to the treatment. Chemotherapy drugs would massively benefit from active targeting of the target cells as it would prevent the main side effects that are caused by this treatment caused by the death of healthy cells. Example of a chemotherapy drug on the market is Taxol (Figure 9).

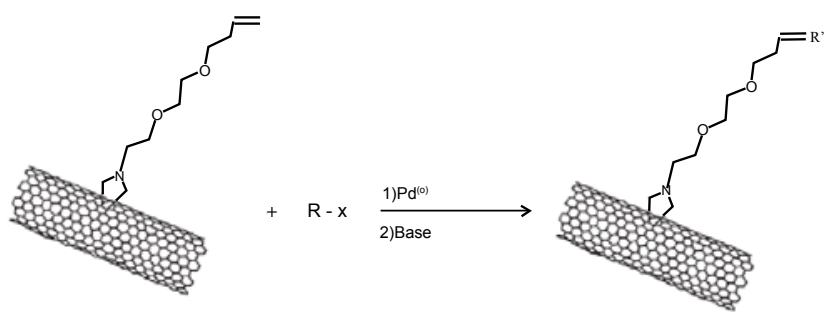

Scheme 4: Via Heck reaction. Adapted from references.31 35 


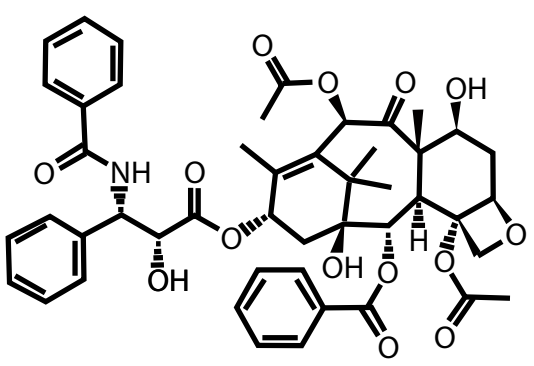

Figure 9: Taxol.

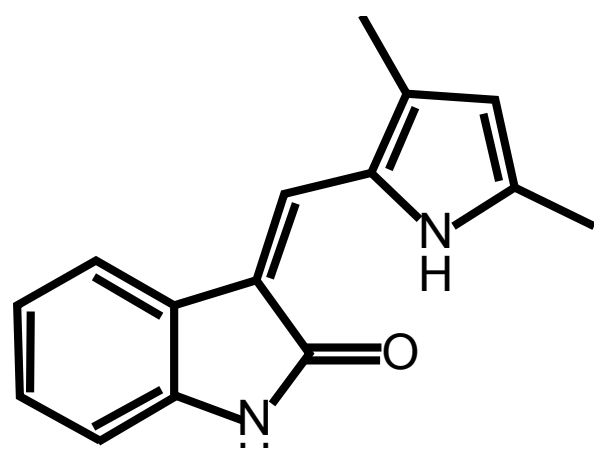

Figure 10: Semaxanib.

In clinical tests at the moment are several drugs designed to target angiogenesis of the tumour cells. This method works by greatly cutting off the tumours blood supply, making the tumour reduce in size and allowing it to be surgically removed. Example of a clinical drug designed to target angiogenesis is Semaxanib (Figure 10)

\section{Radiolabelled and Optically Tagged Targeted Nanoparticles for Brain Imaging}

The vision of Nanotheranostics is that nanocarriers should be used as a diagnostic tool to diagnose diseases. It is doubtful that it is viable for actively targeted nanocarriers to be used for this purpose. This is because it would be a waste of resources to use a nanocarrier for a disease that hasn't been confirmed to be in the body. If the person had already been diagnosed it is possible that it would be helpful to pinpoint the area where the disease is located, and this is the avenue this review will explore.

\section{Molecular imaging}

For this reason diagnostic imaging, in particular radiopharmaceutical imaging is still an area that is crucial to the success of nanocarriers. This is a relatively new discipline that uses probes known as biomarkers to measure biological processes at a molecular level, allowing visualisation of events in a living system without causing any damage. This technique is useful in the diagnosis of cancer, as well as other neurological and cardiovascular diseases. There is a multitude of molecular imaging techniques, in this review we will only focus on a few of them with particular focus on the idea of multi-model imaging probes. The list of the most common of these molecular imaging techniques is in Table 2.

Molecular imaging differs from traditional imaging techniques in that probes known as biomarkers are used to image particular targets. There are several biomarkers that correspond each the imaging technique; this is an area which will need research to find a suitable biomarker for the different applications of nanocarriers. Molecular imaging techniques are different from previous methods of imaging used in medicine. These imaged using the differences in objects or organisms that can be quantified such as density or water content.

Biomarkers are the key element in the operation of all molecular imaging techniques, and therefore these are going to have to be integrated into the design of the nanocarrier. These can be integrated onto a CNT nanocarrier using the coupling chemistry that has already been described. The attachment of this to the nanocarrier design can be seen in Figure 1' as they can be either encapsulated or covalently attached as discussed with reference with drug molecules. Table 2 shows examples of suitable biomarkers for each molecular imaging technique.

Current biomarkers have a very low rate ( 1 in 10000 chance) of reaching their therapeutic targets in vivo [24]. This means that the use of these biomarkers on the actively targeted nanocarrier could be beneficial to both as the active targeting will improve the rate of the biomarker reaching its therapeutic target.

\section{Multimodality imaging}

Molecular imaging techniques currently suffer from a severe limitation as no singular molecular imaging techniques has the detail required for imaging in vivo. This is because the techniques either lack the detail to be used for identifying specific targets or diseases, i.e. fluorescence, or are too detailed and can only scan a small area, i.e. PET. The level of detail presented by each technique is shown in Table 2. Due to these limitations the idea of multi-model imaging has emerged. This is where two imaging techniques are combined, to give both the detail required as well as the overview of the biodistribution of the target or disease. Many different example of multi-model imaging are currently in use, one of the most common is the combination of fluorescence and MRI [24] PET and SPECT could be very useful techniques to use due to their extremely high levels of detail.

Applications of using multimodality imaging with nanomaterials

This utilisation of multi-model molecular imaging with the use of nanocarriers could potentially see many applications, both through the clinical tests and diagnostic potential. Although these applications are not a true diagnostic tool as the vision first implied, it does fulfil the diagnostic purpose in the sense of the examining the efficiency of the therapy.

Visualizing biodistribution in real time: The effectiveness of nanocarriers is dependent on their biodistribution, as it is imperative that they accumulate in the target site. The accumulation of nanocarriers in the incorrect site could lead to negative side effects. Multi-model imaging could facilitate the visualisation of biodistribution in real time,

\begin{tabular}{|l|l|}
\hline Molecular Imaging Techniques & Level of Detail provided \\
\hline Molecular magnetic resonance imaging (MRI) & Molecular \\
\hline Optical Bioluminescence/Fluorescence & Physiological \\
\hline Targeted Ultrasound & Physiological \\
\hline Single Positron Emission Tomography (SPECT) & Molecular (Detailed) \\
\hline Positron Emission Tomography (PET) & Molecular (Detailed) \\
\hline Magnetoresistance probe (MR) & Molecular \\
\hline Information taken from reference [24,36]. & \\
\hline
\end{tabular}

Table 2: Table of Molecular Imaging techniques, relative level of detail and example biomarkers. 


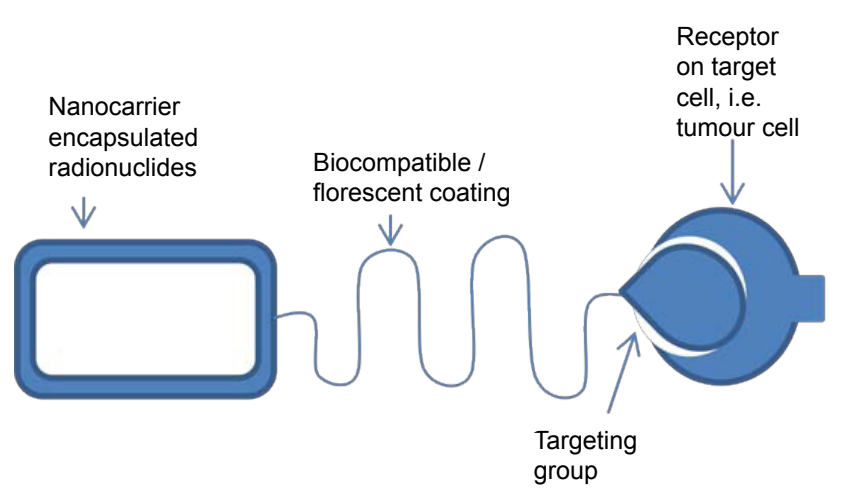

Figure 11: This diagram displays the image of a nanocarrier with a ligand attached and a radionuclide-based imaging agent encapsulated. These allow molecular imaging to take place, and could lead to interesting applications for whole body imaging

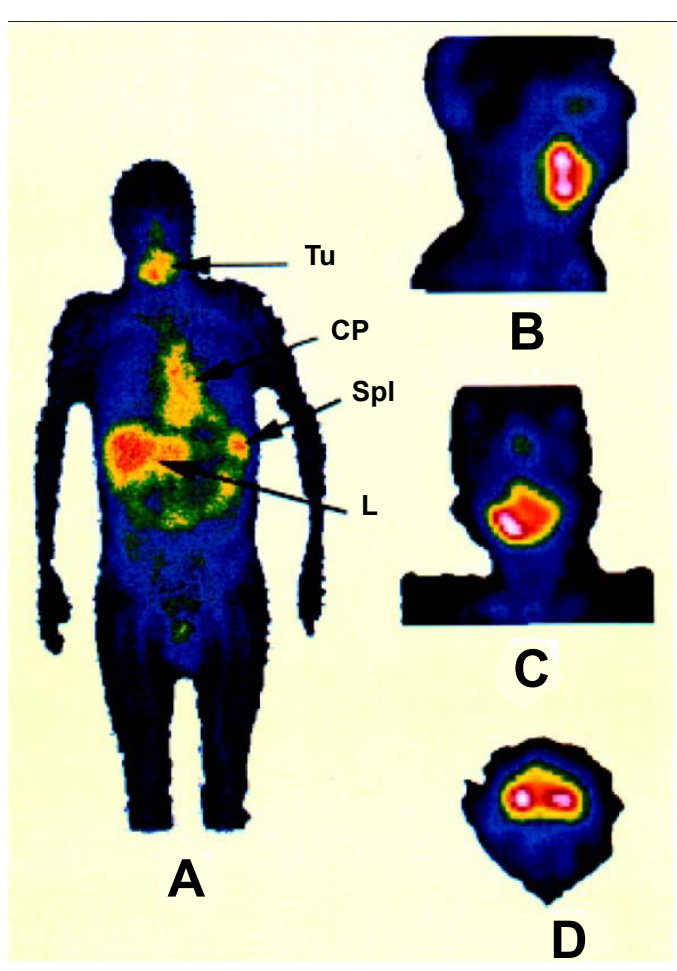

Figure 12: $A, B, C, D$ as it displays the biodistribution of indium-111-labelled PEGylated liposomes. These images were taken 72 hours after injection by SPECT imaging. A shows the overall biodistribution of the nanocarriers, which was targeted at the tumour located in the tongue (Tu). The other places revealed that the nanocarrier collected was in the image was the patient's cardiac pool (CP), liver (L) and Spleen (Spl). B, C, D shows a detailed image of the distribution of Indium-111-labelled PEGylated liposomes in tumour in the tongue. Image adapted from Reference [37].

as all the techniques are non-invasive. This application can be achieved through the use of almost any radionuclides and molecular imaging techniques. This allows a great range of options for the nanocarriers design.

An example of this in action is displayed by Figure $12 \mathrm{~A}$ as it displays the biodistribution of indium-111-labelled PEGylated liposomes. These images were taken 72 hours after injection by SPECT imaging
[38]. This shows how the liposomes have been distributed around the body and collects in different areas. The tumour that was targeted was located in the tongue $(\mathrm{Tu})$, and this is where some of the nanocarriers did collect [38]. The other places revealed that the nanocarrier collected was in the patient's cardiac pool (CP), liver (L) and Spleen (Spl) [38].

Monitoring drug distribution at the target site: Investigation into the overall levels of drug accumulation at the target site is important, but equally important is the ability to visualize and analyse drug distribution at the target site. This allows medical chemists to ensure that there is an equal biodistribution of the nanocarrier throughout the tumour. It is through this method that it was discovered that passively targeted nanocarriers tend to collect in one area of the tumour instead of being equally distributed as mentioned in the introduction. An example of this close imaging is displayed in Figure $12 B$ - $D$ which shows by the distribution of Indium-111-labelled PEGylated liposomes in a tumour in the tongue [38].

Visualizing drug release: The majority of drugs that are attached to nanocarriers are inactive when they are covalently linked [36] Therefore it is vital to ensure that the drugs are being released correctly. Nanocarriers that have imaging capabilities could allow this visualisation to take place. This is important as it is difficult to ensure that the drug have been released in vivo, as the nanocarrier often needs to be homogenised for the drug to be released making imaging difficult once this has occurred [36]. For this technique to function two different imaging agents would be needed, one for when the system is still intact, and one that only produces a signal upon the drugs release. The first would be a form of radionuclide that is attached to or encapsulated in the nanocarrier to allow the monitoring of the nanocarrier with the above applications. As with those applications a wide range of radionuclides can be used. The second would be an agent that is inactive when attached to or encapsulated in the nanocarrier, but is active when it is able to interact with the surrounding biological environment. Examples that have been tried and tested for this use are MR contrast agents (gadolinium and manganese based) as there activation depends on their interaction with surrounding water molecules [39-41].

Facilitating triggered drug release: The applications that have been reviewed so far have been purely based on measuring and reviewing the efficiency of the nanocarriers and the drugs that are attached. Imaging techniques could be used to enhance their effectiveness instead of just being measure. Imaging techniques could allow the optimum release of the drug into the tumorous cells. This could be done if the activation of the drugs release could be activated using an external source. Thereby the imaging techniques could be used to determine the optimum point to release the drug, and then the external source could be used to release the drug. This process could accomplish release of the drug in specific areas of the target site to ensure that only the sites that are in optimum conditions are released. High-intensity focused ultrasound would be an example of a possible external source, as radiofrequency waves could be used for this purpose [36].

Predicting drug responses: Use of multi-model imaging techniques allow additional information to be obtained during the clinical phases of the nanocarrier development. This could be with specific regard to the drugs or nanocarrier that was used. This information allows predictions to be made on the potential effectiveness. This would allow modifications to be made or the investment to be stopped into this specific combination of a drug and a nanocarrier. This could potentially save researchers valuable time and money. This process is referred to as self-regulation; as potential problems can be spotted early 
and corrections can be made. This can be for a wide number of reasons such as effectiveness, toxicity and side effects

Evaluating drug efficacy longitudinally: These applications allow the nanocarrier distribution at the target site to be monitored and altered. This allows extensive studies to be completed to evaluate the effectiveness of targeted drug delivery and to discover the route through the body the nanocarrier took to reach the target site. This will allow medical chemists to better understand and better predict why certain treatments are effective, and why others are not. This is only possible in the cases when all the techniques used (for diagnosis and therapy) are non-invasive, and so can be part of a long term investigation.

\section{Conclusion}

Recently, new strategies to overcome the BBB in order to treat disorders of the CNS (highlighting the role that nanotechnology can play in mediating the transfer of small molecules as well as therapeutic antibodies through this barrier formed by endothelial cells, astrocyte and pericytes) have been developed [42] and reviewed [43].

The ability to combine the diagnosis of disease with therapy using very low doses of the overall drug given to a patient constitutes the original vision of nanotheranostics. Nanomedicines design will lead to improved drug accumulation to the tumour / site of action and a controlled / limited effect on the surrounding healthy tissue. The viability of this vision with regard to diagnosis of disease remains a matter of intense investigations $[44,45]$.

This is especially true if diagnosis is taken in its strictest sense; i.e. the localisation and identification of diseased cells in the body. If diagnosis is interpreting in its broader sense, i.e. the longitudinal study of the therapeutic effects of a nanocarrier drug complex, then there is a great potential as a research tool in this area. This application of imaging is extremely beneficial to both drug design, in both clinical and pre-clinical tests, and allow early identification of otherwise unexpected side effects. The combination of this technology with the new emerging field of precise and active targeting will allow for the development of the nanocarrier drug complex to be efficient and have excellent self-regulation.

Candidates for nanocarriers that can precisely and actively target are only starting to emerge and still have many issues that must be overcome for it to function. The main bottleneck to the design of these nanocarriers will be their ability to cross the BBB.

Encapsulation and targeted delivery will make any drug it carries more effective, reducing the likelihood of side effects, i.e. damage to healthy cells, increased efficiency of the active drug in target site intact and the possibility for externally-triggered release on demand, monitored via imaging of drug delivery. The potential benefits of the design of nanocarriers including those as described above are clear and the risks are similarly under intense public and regulatory scrutiny.

\section{Acknowledgments}

This review is thanks to the support and guidance of Professors Jon Dilworth and Professor Malcolm L.H. Green.

\section{References}

1. Jain MD, Kewal K (2008) The Handbook of Nanomedicine. Humana Press, New Jersey.

2. Peer D, Karp JM, Hong S, FaroKHzad OC, Margalit R, et al. (2007) Nanocarriers as an emerging platform for cancer therapy. Nat Nanotechnol 2: 751-760.

3. Matsumura Y, Maeda $\mathrm{H}$ (1986) A new concept for macromolecular therapeutics in cancer chemotherapy: mechanism of tumoritropic accumulation of proteins and the antitumor agent smancs. Cancer Res 46: 6387-6392.
4. Couvreur P, Vauthier C (2006) Nanotechnology: Intelligent design to treat complex disease. Pharm Res 23: 1417-1450.

5. Yuan F, Dellian M, Fukumura D, Leunig M, Berk DA, et al. (1995) Vascular permeability in a human tumor xenograft: molecular size dependence and cutoff size. Cancer Res 55: 3752-3756.

6. Hobbs SK, Monsky WL, Yuan F, Roberts WG, Griffith L, et al. (1998) Regulation of transport pathways in tumor vessels: role of tumor type and microenvironment. Proc Natl Acad Sci USA 95: 4607-4612.

7. Gottesman MM, Fojo T, Bates SE (2002) Multidrug resistance in cancer: Role of ATP-dependent transporters. Nat Rev Cancer 2: 48-58.

8. Peer D, Margalit R (2006) Fluoxetine and reversal of multidrug resistance. Cancer Lett 237: 180-187.

9. Jain RK (1994) Barriers to drug-delivery in solid tumours. Sci Am 27: 58-65.

10. Mishra B, Patel BB, Tiwari S (2010) Colloidal nanocarriers: a review on formulation technology, types and applications toward targeted drug delivery. Nanomedicine 6: 9-24.

11. Abbott NJ, Patabendige AA, Dolman DE, Yusof SR, Begley DJ (2010) Structure and function of the blood-brain barrier. Neurobiol Dis 37: 13-25.

12. Liu XR, Tu MH, Kelly RS, Chen CP, Smith BJ (2004) Development of a computational approach to predict blood-brain barrier permeability. Drug Metab Dispos 32: 132-139.

13. Bodor N, Buchwald P (2003) Brain-targeted drug delivery: experiences to date. Am J Drug Deliv 1:13-26.

14. Clark DE (2003) In silico prediction of blood-brain barrier permeation. Drug Discov Today 8: 927-933.

15. Begley DJ (2004) Delivery of therapeutic agents to the central nervous system: the problems and the possibilities. Pharmacol Ther 104: 29-45

16. Begley DJ (2004) ABC transporters and the blood-brain barrier. Curr Pharm Des 10: 1295-1312.

17. Eilers M, Roy U, Mondal D (2008) MRP (ABCC) transporters-mediated efflux of anti-HIV drugs, saquinavir and zidovudine, from human endothelial cells. Exp Biol Med 233: 1149-1160.

18. Forster $C$ (2008) Tight junctions and the modulation of barrier function in disease. Histochem Cell Biol 130: 55-70.

19. Giri N, Shaik N, Pan GY, Terasaki T, Mukai C, et al. (2008) Investigation of the role of breast cancer resistance protein (Bcrp/Abcg2) on pharmacokinetics and central nervous system penetration of abacavir and zidovudine in the mouse. Drug Metab Dispos 36: 1476-1484.

20. Dauchy S, Dutheil F, Weaver RJ, Chassoux F, Daumas-Duport C, et al. (2008) ABC transporters, cytochromes $\mathrm{P} 450$ and their main transcription factors: expression at the human blood-brain barrier. J Neurochem 107: 1518-1528.

21. Kamiie J, Ohtsuki S, Iwase R, Unine K, Katsukura Y, et al. (2008) Quantitative atlas of membrane transporter proteins: Development and application of a highly sensitive simultaneous LC/MS/MS method combined with novel in-silico peptide selection criteria. Pharm Res 25: 1469-1483.

22. Sauer I, Dunay IR, Weisgraber K, Bienert M, Dathe M (2005) An apolipoprotein E-derived peptide mediates uptake of sterically stabilized liposomes into brain capillary endothelial cells. Biochemistry 44: 2021-2029.

23. McAfee JG, Neumann RD (1996) Radiolabeled peptides and other ligands for receptors overexpressed in tumor cells for imaging neoplasms. Nucl Med Biol 23: 673-676.

24. Pascu SI, Arrowsmith RL, Bayly SR, Brayshaw S, Hu Z (2010) Towards nanomedicines: design protocols to assemble, visualize and test carbon nanotube probes for multi-modality biomedical imaging. Phil Trans $R$ Soc $A$ 368: 3683-3712.

25. Koo OM, Rubinstein I, Onyuksel H (2005) Role of nanotechnology in targeted drug delivery and imaging: a concise review. Nanomedicine 1: 193-212.

26. Drummond DC, Meyer O, Hong K, Kirpotin DB, Papahadjopoulos D (1999) Optimizing liposomes for delivery of chemotherapeutic agents to solid tumors. Pharmacol Rev 51: 691-743.

27. Sahoo SK, Labhasetwar V (2003) Nanotech approaches to delivery and imaging drug. Drug Discov Today 8: 1112-1120. 
Citation: Chapman M, Pascu SI (2012) Nanomedicines Design: Approaches towards the Imaging and Therapy of Brain Tumours. J Nanomedic Nanotechnol S4:006. doi:10.4172/2157-7439.S4-006

28. Lasic DD, Vallner JJ, Working PK (1999) Sterically stabilized liposomes in cancer therapy and gene delivery. Curr Opin Mol Ther 1: 177-185.

29. Bromberg L (2008) Polymeric micelles in oral chemotherapy. J Control Release 128: $99-112$

30. Bae Y, Diezi TA, Zhao A, KwongS ( 2007) Mixed polymeric micelles for combination cancer chemotherapy through the concurrent delivery of multiple chemotherapeutic agents. J Control Release 122: 324-330.

31. Bianco A, Kostarelos K, Partidos CD, Prato M (2005) Biomedical applications of functionalised carbon nanotubes. Chem Commun (Camb) 571-577.

32. Special Issues on carbon nanotubes (2002) Acc Chem Res 35: 997.

33. LiuY, Wang H (2007) Nanomedicine: Nanotechnology tackles tumours. Nat Nanotechnol 2: 20-21.

34. Georgakilas V, Kordatos K, PratoM, Guldi DM, Holzinger M, et al. (2002) Organic functionalization of carbon nanotubes. J Am Chem Soc 124: 760-761.

35. Clayden J, Nick Greeves, Warren Stuart, Wothers P (2000) Oragnic Chemistry. Oxford.

36. Lammers T, Kiessling F, Hennink WE, Storm G (2010) Nanotheranostics and image-guided drug delivery: current concepts and future directions. Mol Pharm 7: 1899-1912.

37. Harrington KJ, Peters AM, Mohammadtaghi S, Glass D, Epenetos AA (1996) Biodistribution and pharmacokinetics of In-111-labeled Stealth(R) liposomes in patients with solid tumours. J Nucl Med 37: 209-209.
38. Harrington KJ, Mohammadtaghi S, Uster PS, Glass D, Peters AM, et al. (2001) Effective targeting of solid tumours in patients with locally advanced cancers by radiolabeled pegylated liposomes. Clin Cancer Res 243-254.

39. Viglianti BL, Abraham SA, Michelich CR, Yarmolenko PS, MacFall JR, et al. (2004) In vivo monitoring of tissue pharmacokinetics of liposome/drug using MRI: illustration of targeted delivery. Magn Reson Med 51: 1153-1162.

40. Viglianti BL, Michelich C, Abraham S, Colvin OM, Yarmolenko P, et al. (2004 Chemodosimetry of in-vivo tumor liposome/drug concentration using MRI. Int J Radiat Oncol 60: 225-226.

41. Ponce AM, Viglianti BL, Yu D, Yarmolenko PS, Michelich CR, (2007) Magnetic resonance imaging of temperature-sensitive liposome release: drug dose painting and antitumor effects. J Natl Cancer Inst 99: 53-63.

42. Dilnawaz F, Singh A, Mewar S, Sharma U, Jagannathan NR, et al. (2012) The transport of non-surfactant based paciltaxel loaded magnetic nanoparticles across the blood brain barrier in a rat model. Biomaterials 33: 2936-2951.

43. De Rosa G, Salzano G, Caraglia M, Abbruzzese A (2012) Nanotechnologies: a strategy to overcome blood-brain carrier. Curr Drug Metab 13: 61-69.

44. Hu Z, Pantoş, Dan G, Kuganathan, Navaratnarajah, et al. (2012) Interactions Between Amino Acid-Tagged Naphthalenediimide and Single Walled Carbon Nanotubes for the Design and Construction of New Bioimaging Probes. Adv Funct Mater 22: 503-518.

45. Pascu SI, Waghorn PA, Conry T, Lin B, James C, et al. (2009) Metal Ion Controlled Reactivity Book Series: Advances in Inorganic Chemistry 61: 131 178

\footnotetext{
This article was originally published in a special issue, Nanotechnology: Targeted Drug Delivery handled by Editor(s). Dr. Sami M. Nazzal, University of Louisiana at Monroe, USA; Dr. Kytai Troung Nguyen, University of Texas at Arlington, USA
} 